\title{
Survey on Medical Image Segmentation Algorithms
}

\author{
P.Elayaraja ${ }^{1}$, M.Suganthi ${ }^{2}$ \\ Assistant Professor, Department of ECE , Kongunadu College of Engineering and Technology, Trichy, India ${ }^{1}$ \\ Professor, Department of ECE , Mahendra College of Engineering, Salem, India ${ }^{2}$
}

\begin{abstract}
Image segmentation is a process of partition of an image into different objects. There is a significant difference between image enhancement and segmentation. In image enhancement process is to improve the given image quality with respect to image appearance (brightness, contrast, texture).In this segmentation process, the particular portion of a image is highlighted according to the problem defined. Here in this paper we see the performance of the various algorithms for different images.
\end{abstract}

Keywords: Image segmentation, Edge detection, Region based algorithm, Thresholding, Clustering.

\section{I.INTRODUCTION}

Medical image segmentation refers to the process of partitioning observed image data to a serial of nonoverlapping regions. These regions denote different human tissue structures and apply suitable process for accuracy of clinical diagnosis..Generally the basic theory of image segmentation is a process of partitioning a digital image into multiple segments. The goal of segmentation is to simplify and change the representation of an image into something that is more meaningful and easier to analyze.There is large number of applications such as content-based visual information retrieval (CBVIR) system for searching of digital images in large databases. In Object detection, detecting instances of semantic objects of a certain class (such as humans, buildings, road ,forest) In A facial recognition system is a computer application for automatically identifying or verifying a person from a digital image. Fingerprint recognition refers to the automated method of verifying a match between two human fingerprints, for clinical purposes is used to creat a image to reveal ,diagnose or examine the part of human body.

The rest of paper is organized as follows: In section II, briefly describes Edge Detection algorithm. In section III,an overview of Region based algorithm with an example. In section IV, Thresholding Algorithm with an example.In section $\mathrm{V}$, explains the definition and computation steps for clustering method. Section VI concludes this paper.

\section{EDGE DETECTION METHOD}

Edge detection is one of the fundamental steps in image processing, image analysis, image pattern recognition, and computer vision techniques. Generally edge refers to a boundary between two regions in a image. Region boundaries and edges are closely related, since there is often a sharp adjustment in intensity at the region boundaries. The purpose of using edge detectors to identify the points in a digital image at which the image brightness changes sharply or, more formally, has

discontinuities. Discontinuities of intensity pixel refer to either line edge, step edge or ramp edge. If the edge detection step is successful, the subsequent task of interpreting the information contents in the original image may therefore be substantially simplified. Edge detection is a fundamental tool in image processing, machine vision and computer vision, particularly in the areas of feature detection and feature extraction.

There are two main methods for edge detection such as search-based and zero-crossing based. The search-based methods detecting position and direction of edges by estimating gradient magnitude using first order derivative method. In zero-crossing based methods, edge smoothness is estimated by applying Laplacian operator. The most common problems of edge-based segmentation is to find a edge in real border exists.

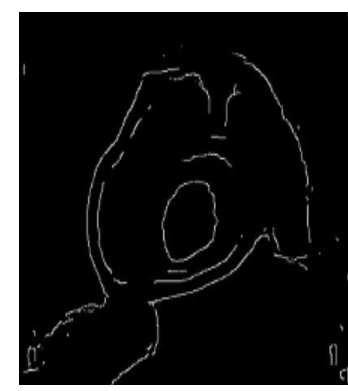

Fig. (1) shows the result of edge detection method

\section{II.REGION-BASED SEGMENTATION METHOD}

Region-based segmentation is a technique for determining the region directly. Region based methods are robust because, Regions cover more pixels than edges and thus have more information available in order to characterize image region. When detecting a region, use texture which is not easy when dealing with edges. Region growing techniques are generally better in noisy images where edges are difficult to detect. 
A. Region split and merge algorithm / Watershed algorithm

i) The given image is subdivided in to four disjoined regions.For example $\mathrm{P}\left(\mathrm{R}_{\mathrm{i}}\right)=$ false.if all pixels have different gray levels in a region.

ii) The Partition process is repeated until to get no more partition.

iii) Merge the neighborhood regions, if they have to share the same pixel intensity.for example $\mathrm{P}\left(\mathrm{R}_{\mathrm{i}} U \mathrm{R}_{\mathrm{i}}\right)=$ true.if two region share same gray level.

The prime advantage of this algorithms are usually less complex, and easy to find region for object detection

B. Watershed algorithm

A watershed is a basin-like landform defined by highpoints and ridgelines that descend into lowerelevations and stream valleys. Watershed algorithm is a region based segmentation techniques image that uses image morphology. Watershed algorithm is an iterative adaptive threshold algorithm.

i) Check the consistent and inconsistent between pair of regions.

ii) For each region in segmentation, check the value of predicate $\mathrm{P}$ with its neighboring regions.

ii) Merge the pairs of neighboring regions whose predicate of $\mathrm{P}$ is true.

The prime advantage of this algorithm, which gives connected components and prior information can be implemented by using markers. The main drawback of this algorithm is fragmentation and over fragmentation problem.

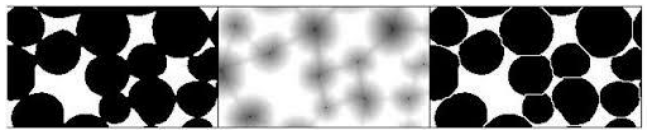

Fig. (2) shows an example of watershed method.

\section{Region growing algorithm}

A simple approach to image segmentation is to start from some pixels (seeds) representing distinct image regions and to grow them, until they cover the entire image. For region growing, need to follow a rule describing a growth mechanism and a rule checking the homogeneity of the regions after each growth step.

i) Select a set of seed points, those who have certain gray level range.

ii) Grow regions only as long as the pixel which has same property. (intensity, gray value)

ii) The above process is repeated until to grow no more region.

The advantages of region growing method are the concept is simple, only small number of seed point enough to grow region. By using this method we can correctly separate the regions that have the same properties and provide original images with have clear edges. The disadvantage of this method is,it consume high computation power and difficult to find good starting point

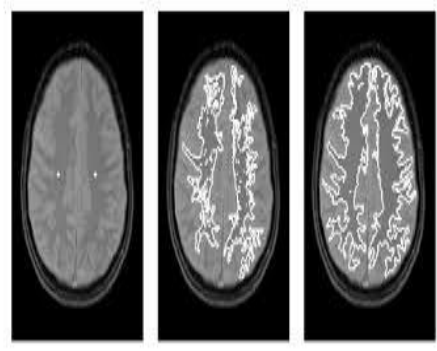

Fig. (3) an example process of region growing method

\section{THREOSHOLDING METHOD / OTSU'S METHOD}

The simplest technique of image segmentation is named the thresholding technique.Thresholding creates binary pictures from grey level ones by turning all pels below some threshold to zero and every one pixel on top of some threshold to at least one. The key of this technique is to pick the edge price or values once multiple-levels area unit elect. In pc vision and image process, Otsu's technique is employed to mechanically perform bar graph shape-based image thresholding, the reduction of a gray level image to a binary image. The rule assumes that the image to be threshold contains 2 categories of pixels or bi-modal bar graph then calculates the optimum threshold separating those 2 categories in order that their combined unfold(intra-class variance) is nominal. The extension of the initial technique to multi-level thresholding is spoken because the Multi Otsu technique. By adjusting threshold a technique, it's attainable to increase the unfold of 1 and reduce the unfold of the opposite. The goal then is to pick the edge that minimizes the combined unfold. It outlines the within-class variance because the weighted add of the variances of every cluster. The advantages of this technique are employed to separate dark and lightweight regions. the most disadvantages of this method no guarantee that the pixels are contiguous because the process consider the pixel intensity only.

1.Separate the pixels into two clusters according to the threshold.

2. Find the mean of each cluster.

3. Square the difference between the means.

4. Multiply by the number of pixels in one cluster times the number in the other.

\section{K-MEANS CLUSTERING METHOD}

The

K-means algorithmic

rule is A repetitive technique that's wont to partition a

picture into $\mathrm{K}$

clusters. It's AN unattended agglomeration algorithmic

rule that classifies the input data points into multiple categories supported their inherent distance from one another. The algorithmic rule assumes that the information options kind a vector area and tries to seek out natural agglomeration in them 
1Pick $\mathrm{K}$ cluster centers, either every which mean cluster approach additionally fails to realize a high way or supported some

degree of accuracy for segmentation and same is that the case with the Dennis Gabor filters technique.

2. Assign every element within the image to the cluster Thus, we are able to conclude that color driven approach that minimizes the space between the element and combined with the therefore the cluster

center.

3. Re-compute the cluster centers by averaging all of the pixels within the cluster.

\section{REFERENCES}

4. Repeat steps two and three till convergence is earned (e.g. no pixels modification clusters).

In this case, distance is that the square or absolute distinction between a element and a cluster center. The distinction is often supported element color, intensity, texture, and placement, or a weighted combination of those factors.

$\mathrm{K}$ are often designated manually, randomly, or by a heuristic. This algorithmic rule is sure to converge, however it's going to not come back the optimum resolution. The standard of the answer depends on the initial set of clusters and therefore the worth of $\mathrm{K}$. This algorithmic rule well appropriate for the applications of optimization drawback. Most k-means-type algorithms need the amount of clusters - - to be laid out in advance, that is taken into account to be one in every of the most important drawbacks of those algorithms

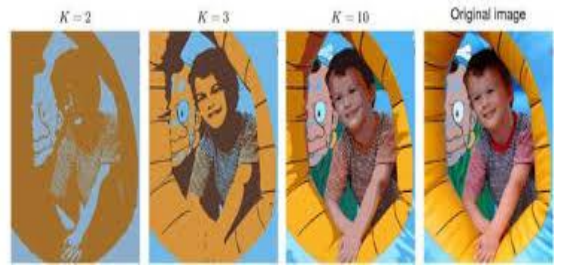

Fig. (5) shows the result of k-means clustering method

\section{V.CONCLUSION}

In Medical image segmentation is a crucial task to find and establish the regions denote totally different human tissue structures. In this paper a comparative study has been performed on the present strategies for the image segmentation. The Segmented image results of varied algorithms square measure compared with a color and intensity options. It has been determined that the Derivate technique yields fruitful results only the complexness is a smaller amount, the complexness of the image may be thought-about on the colored objects.

TheWatershed formula technique additionally suffers

from the downside of no distinct segmentation within the pictures with complexness. The region growing technique additionally suffers from the downside of no distinct segmentation within the pictures with complexness. The ousts technique is not appropriate for multichannel pictures. The k-
[1] Bo Peng,Lei Zhang,and David Zhang 'Automatic Image Segmentation by Dyanamic Region Merging'IEEE Trans on Image proce vol-20,No-12 Dec-2011

[2] S.Kirindis and V. Chatzis, "A Robust Fuzzy Local Information CMeans Clustering Algorithm," IEEE Trans. Image Process. Vol.19, No.5, pp. 1328-1337,May 2010

[3] S Z Beevi and M.M. Sathik, " A robust Segmentation Approach forNoisy Medical Iamges Using Fuzzy Clustering With Spatial Probability," European Journal of Scientific Research, Vol. 41 No. 3 pp.437-451, 2010

[4] L. Liao, D. Y. Wang, F. G. Wang, and L. Yuan, "A fast kernel-based clustering algorithm with application in MRI image segmentation [J]," IEEE International Conference of Intelligent Computing and Intelligent Systems, 2009. ICIS2009. vol. 4, pp. 405-410 (2009).

[5] W. X. Kang, Q. Q. Yang, R. R. Liang ,"The Comparative Research on Image Segmentation Algorithms", IEEE Conference on ETCS, pp. 703-707, 2009

[6] H. Wang, B.J. Zhang, X.Z. Liu, D.Z. Luo, S.B. Zhong, "Image segmentation method based on improved genetic algorithm and fuzzy clustering", Advanced Materials Research vol. 379, pp. 143-144, 2011.

[7] F. A. Peres, F. R. Oliveira, L. A. Neves, and M. F. Godoy, "Automatic segmentation of digital images applied in cardiac medical images," IEEE, 2010.

[8] E. A. Zanaty, S. Aljahdali, N. Debnath, "A kernelized fuzzy c-means algorithm for automatic magnetic resonance image segmentation", Journal of Computational Methods in Science and engineering (JCMSE), pp. 123-136, 2009 